\title{
DESENVOLVIMENTO DE HIDROGÉIS DE POLIETILENO GLICOL E ALGINATO PARA A LIBERAÇÃO CONTROLADA DE BROMELINA
}

\author{
M. F. CAVENAGUE ${ }^{1}$, P. G. MAZZOLA ${ }^{2}$; B. ZANCHETTA ${ }^{3}$; E. B. TAMBOURGI ${ }^{3}$; \\ E. SILVEIRA ${ }^{1}$ \\ ${ }^{1}$ Universidade Federal de Uberlândia, Instituto de Genética e Bioquímica \\ 2 Universidade Estadual de Campinas, Faculdade de Ciências Médicas \\ ${ }^{3}$ Universidade Estadual de Campinas, Faculdade de Engenharia Química \\ E-mail para contato: maria.fernanda1994@yahoo.com.br
}

\begin{abstract}
RESUMO - Os hidrogéis têm sido amplamente utilizados para inúmeras aplicações biológicas, médicas e tecnológicas, por possuírem algumas características peculiares, como atoxicidade; capacidade de intumescer em água e fluído biológicos; consistência elastomérica, o que minimiza o atrito entre tecidos e o hidrogel; alta permeabilidade, facilidade de obtenção em diferentes formas; incorporação e liberação controlada de fármacos de diferentes polaridades. Os hidrogéis podem ser formados por polímeros naturais, naturais modificados ou sintéticos. A bromelina é uma proteína encontrada nos vegetais da família bromeliaceae, e possui diversos usos na produção de fármacos, na indústria alimentícia, no tratamento de distúrbios digestivos, feridas e inflamações, no preparo de colágenos hidrolisados, nas indústrias têxteis, para amaciamento de fibras e também na produção de detergentes. O estudo visou a formulação de hidrogéis a base de polietileno glicol (PEG) e alginato, avaliando a absorção de água nos hidrogéis formulados e analisando a incorporação e liberação de bromelina em diferentes temperaturas para a liberação controlada de drogas, encontrando como formulação ideal uma concentração de $2 \%$ de PEG e $2 \%$ de alginato em pH 7 e temperatura ideal para liberação de bromelina, em concentrações de $0,2 \%, 25^{\circ} \mathrm{C}$.
\end{abstract}

\section{INTRODUÇÃO}

Hidrogéis podem ser definidos como sistemas hidrofílicos formados por dois ou mais componentes unidos por ligações covalentes e/ou eletrostáticas dispostos em uma ou mais redes tridimensionais estruturadas envoltas por moléculas de um determinado solvente, geralmente água.

Segundo Moura (2005) os hidrogéis possuem algumas características peculiares, por exemplo: atoxicidade; capacidade de intumescer em água e fluído biológicos; consistência elastomérica, o que minimiza o atrito entre tecidos e o hidrogel; alta permeabilidade, o que permite o fluxo de fluídos corpóreos pelo hidrogel; facilidade de obtenção em diferentes formas; incorporação e liberação controlada de fármacos de diferentes polaridades. 
Os hidrogéis podem ser formados por polímeros naturais (Boucard et al., 2007) tais como ácido hialurônico, alginato, quitosana; por polímeros naturais modificados (Coviello et al., 2007), por exemplo dextrana reticulada, galactomanana metacrilatada; e por polímeros sintéticos (Sedláková et al., 1998) tais como polietileno glicol (PEG), poliacrilamida (PAAm), poli(hidroxietil metacrílico) (HEMA), poli( $N$-isopropilacrilamida) (PNIPAAm), entre outros.

Hidrogéis sintéticos a base de PEG possuem alto equilíbrio de conteúdo de água, o que deve fornecer transporte rápido de moléculas pequenas através do gel. O ambiente aquoso dos hidrogéis a base de PEG é apropriado para a imobilização de várias biomoléculas, tais como: proteínas, ácidos nucléicos, e até células inteiras. Além disso, hidrogéis a base de PEG têm demonstrado serem biocompatíveis (Lee et al., 2007). O que os tornam bastante eficazes nas aplicações farmacêuticas, biológicas e bioquímicas, principalmente na liberação controlada de drogas.

Dentre os vários polímeros de origem natural existentes, destaca-se o alginato, o qual é obtido a partir do ácido algínico, encontrado nas paredes das células de algas marrons (Wang e Spencer, 1998). O hidrogel a base de alginato é comumente usado para aplicações endovasculares, no transplante de células, imobilização de enzimas, entre outras (Li et al., 2002). Sua aplicação na área biomédica deve-se a comprovada biocompatibilidade, hidrofilicidade e baixo custo (Hurteaux et al., 2005).

A bromelina é um conjunto de isoenzimas proteolíticas encontradas nos vegetais da família Bromeliaceae, da qual o abacaxi (Ananas comosus) é o mais conhecido (Borracini, 2006). Esta proteína tem diversos usos, todos baseados em sua atividade proteolítica. A sua importância econômica está relacionada com a produção de fármacos e sua utilização na indústria alimentícia (na clarificação de cervejas, na fabricação de queijos, no amaciamento de carnes, no preparo de alimentos infantis e dietéticos, entre outros), no pré-tratamento de soja, no tratamento de distúrbios digestivos, feridas e inflamações, preparo de colágenos hidrolisados, no tratamento do couro, nas indústrias têxteis, para amaciamento de fibras e também na produção de detergentes (Draetta e Giacomelli, 1993).

Um grande número de estudos clínicos sustenta o uso de extratos contendo bromelina para o tratamento anti-inflamatório. Além da atividade anti-inflamatória, a bromelina possui ação comprovada na inibição de agregação plaquetária (Rowan et al., 1990); atividade fibrinolítica (Taussig e Nieper, 1979); antitumoral (Jeung e Foster, 1980); modulação de citocininas e do sistema imune (White et al., 1988); adjuvante na absorção de drogas (Morita et al., 1979); propriedades mucolíticas; assistência da digestão; adjuvante da cicatrização; e aumento da capacidade cardiorrespiratória (Silveira, 2007).

\section{MATERIAIS E MÉTODOS}

\subsection{Preparo dos hidrogéis}

Polietileno glicol (PEG) e alginato foram misturados em tampão fosfato de sódio com diferentes $\mathrm{pH}$. A solução foi colocada em contato com uma solução de cloreto de cálcio em isopropanol em uma concentração de $10 \%$, a fim de promover a polimerização do gel. Em 
seguida, a solução foi lavada com água destilada, a fim de remover o cloreto de cálcio. Foram testadas diferentes concentrações de PEG e alginato.

\subsection{Absorção de água}

Para os testes de absorção de água o hidrogel foi seco em estufa por 24 horas e pesado em balança analítica, em seguida foi hidratado com água destilada e pesado novamente. Para estimar a porcentagem de água absorvida dividiu-se o peso do gel seco pelo peso do gel hidratado.

\subsection{Incorporação da bromelina no hidrogel}

Primeiramente, o hidrogel foi preparado nas concentrações de PEG e alginato que melhor absorveram água, juntamente com a bromelina em concentrações $0,1 \%$ e $0,2 \%$. Em seguida, o hidrogel foi polimerizado em solução de $10 \%$ de cloreto de cálcio em isopropanol. Após a polimerização do hidrogel, este foi liofilizado e em seguida incubado em tampão PBS a temperaturas de $25^{\circ} \mathrm{C}, 37^{\circ} \mathrm{C}$ e $39^{\circ} \mathrm{C}$.

Posteriormente, colheu-se amostras nos tempos 0,1,2,3,4,5,10,20 e 30 minutos para realizar os testes de atividade da proteína. A atividade da bromelina foi analisada pela absorbância no espectrofotômetro.

\section{RESULTADOS E DISCUSSÕES}

Foram preparados hidrogéis com diferentes porcentagens de PEG e alginato e em diferentes $\mathrm{pH}$ como apresentado na tabela 1 .

Tabela 1 - Concentrações de PEG e alginato em diferentes $\mathrm{pH}$

\begin{tabular}{cccc}
\hline & PEG (\%) & Alginato (\%) & pH \\
\hline $\mathbf{4}$ & 3,0 & 3,0 & 6,0 \\
$\mathbf{9}$ & 2,0 & 2,0 & 7,0 \\
$\mathbf{1}$ & 1,0 & 1,0 & 6,0 \\
$\mathbf{2}$ & 3,0 & 1,0 & 6,0 \\
$\mathbf{3}$ & 1,0 & 3,0 & 6,0 \\
$\mathbf{5}$ & 1,0 & 1,0 & 8,0 \\
$\mathbf{7}$ & 1,0 & 3,0 & 8,0 \\
$\mathbf{8}$ & 3,0 & 3,0 & 8,0 \\
$\mathbf{1 1}$ & 2,0 & 2,0 & 7,0 \\
\hline
\end{tabular}

Os resultados da absorção de água para as diversas formulações de hidrogel estão expostos na tabela 2 .

Tabela 2 - Absorção de água e degradação do hidrogel

\section{Gel seco (g) \\ Gel hidratado (g) \\ Porcentagem (gel seco/gel} hidratado) $\%$ 


\begin{tabular}{cccc}
\hline $\mathbf{4}$ & 0,4241 & 0,8878 & 2,093 \\
$\mathbf{9}$ & 0,3897 & 2,6793 & 6,875 \\
$\mathbf{1}$ & 0,2682 & 0,9142 & 3,408 \\
$\mathbf{2}$ & 0,3973 & 0,7624 & 1,918 \\
$\mathbf{3}$ & 0,4674 & 2,5261 & 5,404 \\
$\mathbf{5}$ & 0,4537 & 2,836 & 6,250 \\
$\mathbf{7}$ & 0,6489 & 4,345 & 6,695 \\
$\mathbf{8}$ & 0,7911 & 4,806 & 6,075 \\
$\mathbf{1 1}$ & 0,4502 & 3,0822 & 6,8462 \\
\hline
\end{tabular}

Todas as formulações dos hidrogéis polimerizaram e absorveram água sem perder sua conformação conforme mostra a figura 1. Porém, os hidrogéis nas concentrações de 2:2 em pH 7 e 3:3 em pH 8 foram os mais eficazes na absorção de água, ou seja, eles conseguiram absorver uma grande quantidade de água mantendo a sua formação polimérica. Assim, a taxa de absorção desses hidrogéis foram altas indicando que nessas concentrações, eles são biocompatíveis e propícios no desenvolvimento do hidrogel imobilizado com a bromelina. $\mathrm{O}$ hidrogel utilizado nos métodos seguintes foi o de concentração de $2 \%$ de PEG e $2 \%$ de alginato em pH 7 .

Figura 1 - Hidrogel Polimerizado.

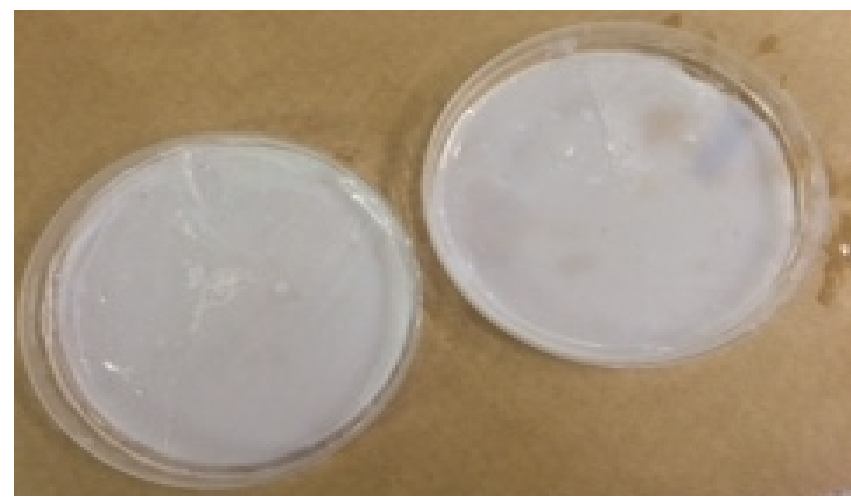

Os resultados obtidos pela absorbância no espectrofotômetro para as diferentes concentrações de bromelina estão apresentados nas tabelas 3 e 4.

Tabela 3 - Atividade da Bromelina $0,1 \%$

\begin{tabular}{cccc}
\hline Bromelina 0,1\% & \multicolumn{3}{c}{} \\
\hline & $25^{\circ} \mathrm{C}$ & $37^{\circ} \mathrm{C}$ & $39^{\circ} \mathrm{C}$ \\
\cline { 2 - 4 } t0 & 0,055 & 0,025 & 0,022 \\
t1 & 0,041 & 0,022 & 0,023 \\
t2 & 0,037 & 0,034 & 0,020 \\
t3 & 0,042 & 0,017 & 0,025 \\
$\mathbf{t 4}$ & 0,045 & 0,017 & 0,026 \\
$\mathbf{t 5}$ & 0,047 & 0,014 & 0,023 \\
$\mathbf{t 1 0}$ & 0,065 & 0,013 & 0,032 \\
$\mathbf{t 2 0}$ & 0,067 & 0,021 & 0,029 \\
\hline
\end{tabular}




\section{t30}

0,079

0,036

0,027

Tabela 4 - Atividade da Bromelina 0,2\%

\begin{tabular}{cccc}
\hline Bromelina 0,2\% & \multicolumn{3}{c}{} \\
\hline & $25^{\mathbf{0}} \mathrm{C}$ & $37^{\mathbf{0}} \mathrm{C}$ & $39^{\mathbf{0}} \mathrm{C}$ \\
$\mathbf{t 0}$ & 0,001 & 0,043 & 0,005 \\
$\mathbf{t 1}$ & 0,017 & 0,054 & 0,009 \\
$\mathbf{t 2}$ & 0,026 & 0,058 & 0,016 \\
$\mathbf{t 3}$ & 0,048 & 0,064 & 0,028 \\
$\mathbf{t 4}$ & 0,037 & 0,072 & 0,030 \\
$\mathbf{t 5}$ & 0,048 & 0,066 & 0,031 \\
$\mathbf{t 1 0}$ & 0,038 & 0,102 & 0,046 \\
$\mathbf{t 2 0}$ & 0,070 & 0,114 & 0,110 \\
$\mathbf{t 3 0}$ & 0,103 & 0,108 & 0,023 \\
\hline
\end{tabular}

Com base nos resultados obtidos, a temperatura que a proteína Bromelina em ambas as concentrações teve maior atividade nos determinados tempo foi a de $25^{\circ} \mathrm{C}$. A concentração de maior atividade foi de $0,2 \%$ de Bromelina. Este resultado indica que a Bromelina foi incorporada com eficiência no hidrogel e desempenha sua atividade proteica nas determinadas concentrações e temperaturas.

\section{CONCLUSÃO}

Os resultados do trabalho sugere que os hidrogéis a base dos polímeros sintético PEG e natural alginato sejam eficientes no processo de entrega controlada de drogas, uma vez que o mesmo apresentou alta taxa de biocompatibilidade e eficiência na incorporação da bromelina, a qual possui diversas aplicações terapêuticas.

\section{REFERÊNCIAS}

BORRACINI, H. M. P. Estudo do processo de extração da bromelina por micelas reversas em sistema descontínuo. Dissertação (Mestrado em Engenharia Química). Universidade Estadual de Campinas, Campinas, p.76, 2006.

BOUCARD, N.; et al. Biomaterials, ed. 28, p. 3478, 2007.

COVIELLO, T.; et al. J. Controlled Release, 119, p.5, 2007

DRAETTA, I. S.; GIACOMELLI, E. J. Ocorrência da bromelina e cultivares de abacaxizeiro. Colet. ITAL, Campinas, v.23, n.1, p.44-55, 1993.

HURTEAUX, R.; et al. Eur. J. Pharm. Sci, ed.24, p.187, 2005. 
JEUNG, A.; FOSTER, S. Encyclopedia of common natural ingredients used in foods, drugs, and cosmetics. New York, USA: John Wiley \& Sons, 1980.

LEE, W.; LEE, T.G.; KOH, W. Grafting of Poly(acrylic acid) on the Poly(ethylene glycol) Hidrogel Using Surface-initiated Photopolymerization for Covalent Immobilization of Collagen. Journal of Industrial Engineering and Chemistry, v. 13, n.7, p. 1195-1200, 2007.

LIN, C.; METTERS, A. T. Adv. Drug Delivery Rev., ed.58, p.1379, 2006.

MORITA, A.H.; UCHIDA, D.A.; TAUSSIG, S.J. Chromatographic fractionation and characterization of the active platelet aggregation inhibitory factor from bromelain. Arch Inter Pharm Ther, v.239, p.340-350, 1979.

MOURA, M. R. de. Caracterização de Matriz Polimérica de Hidrogel Termosensível Sintetizada a Partir de Alginato-Ca2+e Poli(N-isopropil acrilamida), do Tipo IPN e Semi-IPN. 97 f. Dissertação (Mestrado) - Universidade Estadual de Maringá, Maringá, 2005.

ROWAN, A.D.; BUTTLE, D.J.; BARRETT, A.J. The cysteine proteinases of the pineapple plant. Biochem. J., v.266, p.869-875, 1990.

SEDLÁKOVÁ, Z.; BOUCHAL, K.; ILAVSKÝ, M. Polymer Gels and Networks,ed.6, p. 163, 1998.

SILVEIRA, E. Purificação e Caracterização de Bromelina a Partir do Extrato Bruto de Ananas comosus por Adsorção em Leito Expandido. (Dissertação de Mestrado). Faculdade de Engenharia Química, Universidade Estadual de Campinas, Campinas, p. 74, 2007.

TAUSSIG, S.J.; NIEPER, H.A. Bromelain: its use in prevention and treatment of cardiovascular disease, present status. J IAPM, v.6, p.139-151, 1979.

WANG, X.; SPENCER, G. Polymer, ed.39, p.2759, 1998. 